ideas, but it has the tremendous advantage that the reader can dive into any section of interest and feel at ease.

The second part of the book, on diseases, is a random walk through five different genetic conditions. This allows Reilly to explore different aspects of medicine, different disease aetiologies, different modes of therapy, and the complex ethics and sociology of conditions such as deafness, in which non-hearing parents may actually want to have non-hearing children. Missing from Reilly's treatment is a deeper look at genetic mechanisms. Trinucleotide repeats are introduced as more or less incidental to Huntington's disease, rather than as part of a set of more than a dozen disorders with an astoundingly similar underlying mutation mechanism that involves rapid changes in highly unstable stretches of DNA.

The third part of the book is an investigation of dogs, cats, mice, corn and rice, what genetics has meant for them, and what these organisms have meant for the development of modern genetics. Reilly's treatment of corn is typical of his approach to other organisms. He writes about corn's place in the human economy, its domestication and breeding, and what we have learned about its genetics. He then analyses some of the work on genetically modified corn, addressing a topical issue that could have profoundly disturbing implications for ecology and food safety - or that could lead to fantastic gains in the war on hunger. Reilly does not ignore the arguments against the use of genetically modified foods. He even sympathizes with those who fear multinational agriculture companies taking over the world at the expense of small farmers. But there's no question how Reilly feels about this technology. "I side squarely with those who favor the rapid, safe development of genetically modified groups. I think it could be the most important positive event for the environment in history."

The final section deals more specifically with the implications of genetic and reproductive technologies for our society. Reilly addresses not only the obvious topics such as stem-cell biology, preimplantation genetic diagnosis, and forensics, but also what we can learn about history through genetics (tracing US president Thomas Jefferson's genes, for example), and how genetics has come to pervade our art and language.

For the geneticist, Reilly presents a balanced, positive view of ethical and social issues in genetics, and an entertaining background in history, geography and economics, and the way these fields interface with modern genetics and genomics. I've often tried to convince my colleagues across campus that genetics should be a part of every undergraduate's education. No book makes this case more clearly than The Strongest Boy in the World.

Michael A. Goldman is in the Department of Biology, San FranciscoState University, San Francisco, California 94132-1722, USA.

\section{A glimpse of the early Universe}

Chasing Hubble's Shadows: The Search for Galaxies at the Edge of Time by Jeff Kanipe

Hill \& Wang: 2006.224 pp. \$24

\section{Patrick Petitjean}

Can beauty save the world? Anyone who reads Chasing Hubble's Shadows by Jeff Kanipe may be able to find an answer in the book's images of a tiny part of the sky, taken by the Hubble Space Telescope. They reveal thousands of galaxies, the "Hubble's shadows" of the title, at different stages of their evolution. The images portray breathtaking beauty surrounded by silence.

The book is about the beauty of the heavens as revealed by Hubble, the questions such images raise, and the explanations astronomers have proposed, along with their doubts and hopes. How did astronomers become interested in galaxies? How can galaxies be classified? How are the most remote of them detected? How can their formation and evolution be explained? The book is much more than a description of the farthest images ever taken. It is also a vivid description of how astronomers work and how ideas develop. It is a virtual trip into the complex realm of dark matter and dark energy, but also a real trip through the Moon-like landscape of the Mauna Kea summit in Hawaii where the world's biggest telescopes are located. This is an exciting time, with astronomers facing challenges similar to those that confronted Galileo when he discovered the satellites of Jupiter. Astronomers are trying to understand the physical processes at play in the early Universe.

Kanipe has written a narrative rather than an analysis. He reports discussions with dozens variety of subjects relevant to the early Universe and to the formation and evolution of galaxies. The writing is uneven and a few topics are discussed several times, but isn't the art of saying things several times without bothering the reader part of pedagogy? The resulting mixture of journalistic and scientific styles certainly keeps the reader excited.

The book will not help readers understand physics from first principles, or discover a theory that can make sense of the little information we have on our Universe. Rather, it provides an opportunity to meet people and ideas. This is an entertaining way to approach the relation between the big scientific questions and the emotions that overcome astronomers during their research. We hear of their nightmares, their doubts, their patience, their scepticism and the risks they take.

It is difficult to define the target audience for this book. There are sometimes too many inappropriate technical details for a general reader and, probably driven by his enthusiasm, the author also goes too fast or too far. For example, it may be unrealistic to assume that general readers know what a graviton is, or why it may be interesting to follow up on radio galaxies, and specialists will learn little too. Nonetheless, if general readers are happy to leave a few technical details unexplained, they will be able to enjoy this entertaining approach to the mysteries of our Universe. Anyone curious about the mechanisms of our world will be thrilled by the book.

Few would disagree with the remarks here concerning the lack of funding, although these are hardly unique to astronomy. Kanipe also questions the political choices being made, of astronomers and their explanations of a

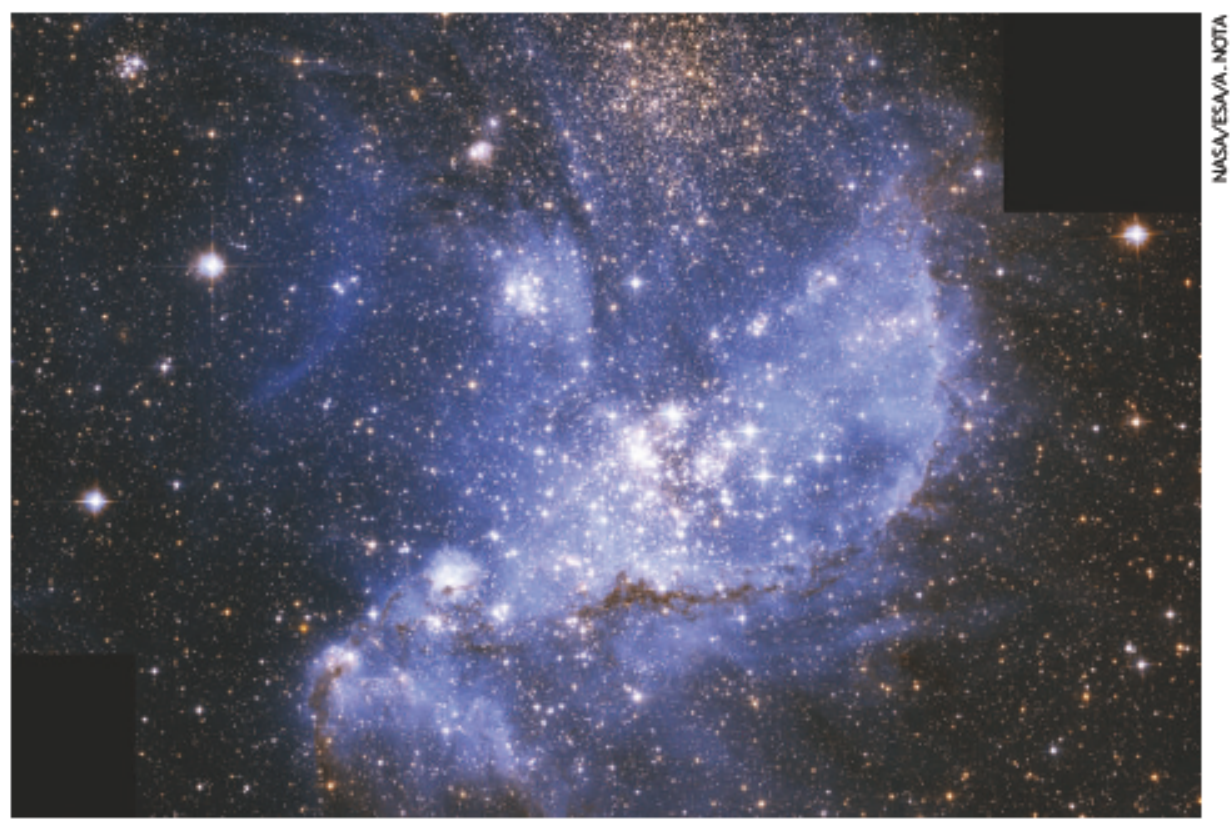

The Hubble Space Telescope has identified infantstars in the Small Magellanic Cloud galaxy. 
such as the US plan to visit the Moon and Mars. It would have been more appropriate to question the place of science and basic research in our society. This has to be carefully argued to convince a broad audience, but I am afraid he is preaching to the converted.
The main aim of the book is to describe what astronomers have learned so far about the first stars and galaxies in the early Universe, but it does much more than this. Given the huge strides being made in exploring the Universe, physicists are having to find new and different ways of explaining what they see. This creates the feeling that we have reached the end of the beginning and are looking ahead.

Patrick Petitjean is at the Institut d'Astrophysique de Paris, 98 bis Boulevard Arago, 75014 Paris, France.

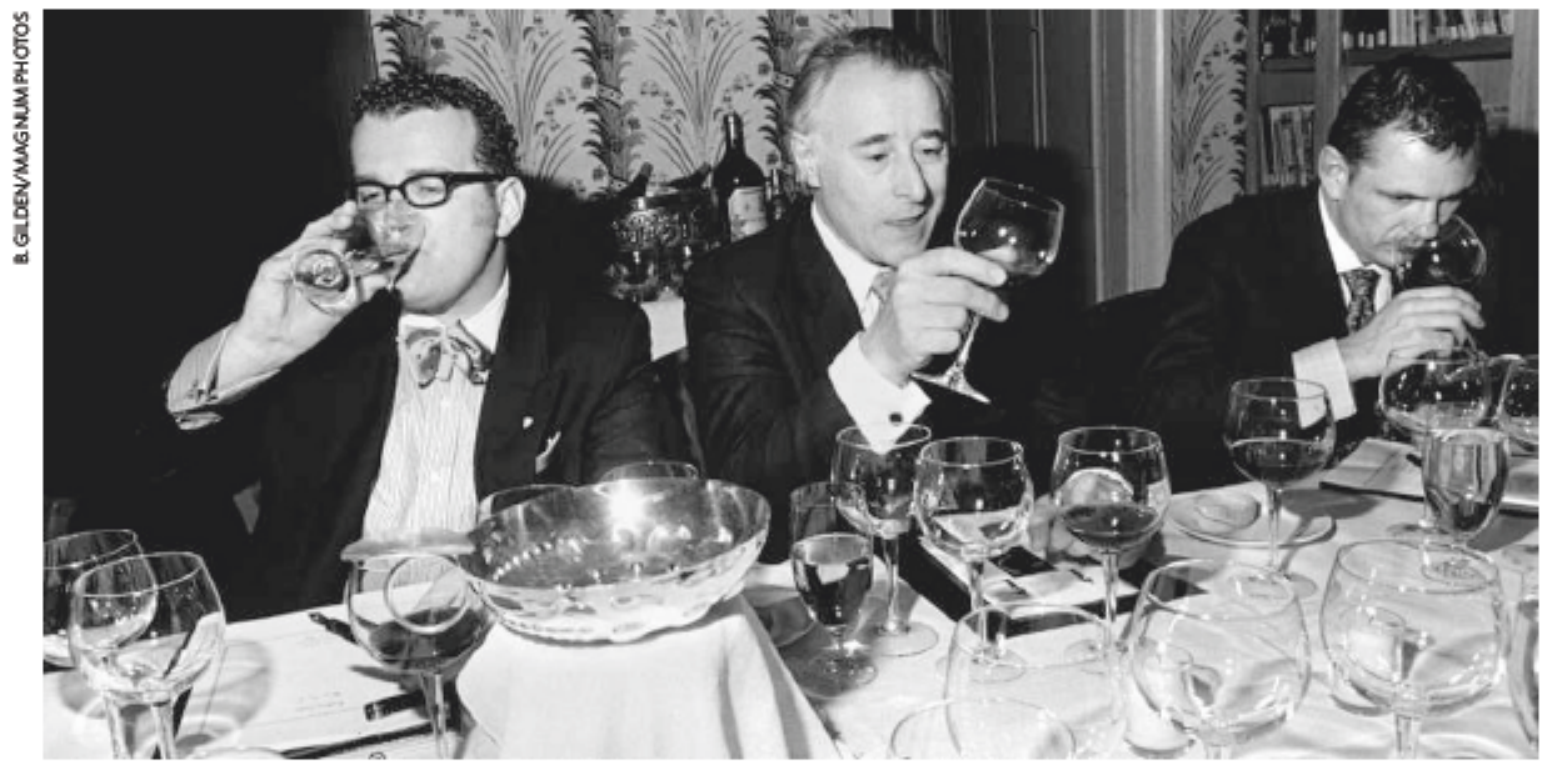

Glass distinction:it's all in the flavour, the colour and the bouquet for those who wish to enjoy wines or identify them in a 'blind' tasting.

\section{A question of taste}

Molecular Gastronomy: Exploring the Science of Flavor

by Hervé This (transl. by M. B. DeBevoise) Columbia University Press: 2006. 360 pp. $\$ 29.95$

\section{John Piggott}

In the introduction to this book, Herve This says what he means by 'molecular gastronomy', explains its origins and carefully differentiates it from food science. In essence, he defines molecular gastronomy as the science (physics, chemistry and biology) of enjoying food. However, insofar as it is science applied to food, it is simplest to regard it as a branch of food science, concerned particularly, although not exclusively, with the transformation of ingredients to foods and their subsequent consumption. He applies it especially to culinary, rather than industrial, processes.

Molecular Gastronomy is an anthology of columns from Pour la Science (the French edition of Scientific American), mostly published in the 1990s. The short chapters are organized into four sections. The first part describes investigations and explanations of traditional cooking methods, and the second covers the physiology of flavour (mostly taste). The third and longest part is composed largely of discussions of journal articles and news stories, with most being explanations of how traditional products work. Finally, applications of recent science and technology are discussed to suggest new products derived from existing ones. At the end there is a glossary, a list of further reading and a good index.

One or two of the explanations in the first section are not convincing but would make a good starting point for anyone wanting to take them further. Elsewhere the descriptions and explanations of recent science are clear and coherent but uncritical. For example, he cites a statistical analysis of some Scotch whisky tasting data that demonstrates only that if you use inappropriate data in a statistical analysis you get meaningless results. There seems to be disproportionate attention paid to alcoholic beverages, including a chapter about warming and chilling wine. This did not go far enough for me; there is no help with practical problems, such as how long you should you leave a bottle of white wine or champagne in the freezer to cool it quickly, and whether it matters. Or how long a bottle of red wine left overnight at $-5^{\circ} \mathrm{C}$ and needed for lunch should be left in the microwave oven and at what power?

Most of the work discussed was done in France, but maybe only laboratories there work on products of interest to a French audience, or perhaps they are better at publicity. It is both a weakness and a strength of this book that it is French. I cannot imagine anyone being funded to do this work in Britain or the United States, where there is less interest in food, but at least someone is doing it. Surely no one outside France cares whether the yolk is exactly in the middle of a boiled egg, and the English-speaking world, which is presumably the target for this edition, probably cares even less than the rest. In fact it is hard to see where the market for this book would be. Reading these intelligent but light-hearted columns in a monthly magazine is one thing, but buying and reading the book is quite another. Many of these stories date from 10 to 15 years ago, which is regarded as history by several of my colleagues, and even in food science the work is likely to have been overtaken by events.

The translation is generally very good, and the book makes a worthy attempt to explain the science to non-special ist readers. There are a few mistakes, but not important ones. For example, it refers to Brussels sprouts as a root vegetable - have I been eating the wrong bit all these years? In any case, the original sources are listed as 'further reading', although the individual chapters do not cite the references.

There is too much science to make this a mass-market book, and it is certainly not for the specialist food scientist. It would make a good present, perhaps, for a 'foodie' scientist, and I would put it on the reading list for an introductory food-science course, as it might interest some of the students.

John Piggott is in the Department of Bioscience, University of Strathclyde, Glasgow G1 1XW, UK.

\section{Correction}

The Science in Culture "Home from home"

(Nature 441,816; 2006) by Colin Martin should

have said that Benjamin Franklin died in

Philadelphia, rather than at Passy near Paris. 